\title{
Antibiotic Prophylaxis for VCUG: A Cross-Sectional Observational Study
}

\author{
Suprita Kalra ${ }^{1}$ (D) $\cdot$ S. K. Dey ${ }^{2} \cdot$ R. Shankar ${ }^{2} \cdot$ Madhuri Kanitkar $^{1}$
}

Received: 14 May 2019 / Accepted: 20 December 2019 / Published online: 8 January 2020

(C) Dr. K C Chaudhuri Foundation 2020

To the Editor: Standard guidelines exist for doing voiding cystourethrogram (VCUG) in children with recurrent urinary tract infections (UTI) and children with Congenital anomalies of kidney and urinary tract (CAKUT) [1]. There is no consensus on whether children should be routinely given antibiotics before VCUG or not to prevent UTI. We therefore conducted this study with the aim to determine the frequency of UTI following voiding cystourethrography (VCUG) under strict asepsis with no additional antibiotic prophylaxis for the procedure. All children aged 0 to 12 y undergoing VCUG were enrolled after informed consent. These children were put on antibiotic prophylaxis (sulphamethoxozole + trimethoprim or cephalexin) while awaiting VCUG as per the ISPN guidelines [1]. Children undergoing early VCUG while on full dose of antibiotics for treatment of UTI were excluded. All children underwent sterile bladder catheterisation at Pediatric ward by the duty residents. They were advised to report if they developed fever or dysuria or both within $7 \mathrm{~d}$ and urine microscopic examination and culture was done. UTI was diagnosed using standard definitions $[2,3]$. Sixty-one children (35 boys/26 girls) underwent VCUG from January through December 2018 (12 mo). Five children were excluded as VCUG was done while they were on full dose of antibiotics for UTI. Forty-seven children $(83.92 \%, 13$ neonates, 24 infants and 10 between 1 and $5 \mathrm{y}$ of age) were already on antibiotic prophylaxis. The age at the time of VCUG ranged from $6 \mathrm{~d}$ to 128 mo. VCUG showed posterior urethral valves (PUV) in 3, primary vesicoureteric reflux (VUR) in 24 (16 grade I-III and 8 grade IV-V) and neurogenic bladder in 4 . The remaining had

Suprita Kalra

kalrasuprita@gmail.com

1 Department of Pediatrics and Pediatric Nephrology, Command Hospital (SC), AFMC, Pune, India

2 Department of Pediatric Surgery, Command Hospital (SC), AFMC, Pune, India normal VCUG. Only 2 (3.57\%) children (none of whom were on antibiotic prophylaxis as they had an apparently normal ultrasound KUB) developed fever and /dysuria after VCUG. Both had E. coli $>10^{5} \mathrm{CFU}$ on urine culture and grade IV VUR.

Our study elegantly brings out that risk of culture positive UTI post VCUG when bladder catherization is done under strict asepsis is minimal in children already on prophylactic antibiotics and therefore additional antibiotics before VCUG can be avoided. Larger prospective studies would be however required to prove this conclusively.

\section{Compliance with Ethical Standards}

Conflict of Interest None.

\section{References}

1. Sinha A, Bagga A, Krishna A, et al; For the Indian Society of Pediatric Nephrology. Revised guidelines on management of antenatal hydronephrosis. Indian Pediatr. 2013;50:215-31.

2. Vijayakumar M, Kanitkar M, Nammalwar BR, et al. Revised statement on management of urinary tract infections. Indian Pediatr. 2011;48:709-17.

3. NICE guideline-Urinary tract infection in under 16s: diagnosis and management. Available at: www.nice.org.uk/guidance/cg54/chapter/ 1-Guidance\#imaging-tests. Accessed 01 Feb 2018.

Publisher's Note Springer Nature remains neutral with regard to jurisdictional claims in published maps and institutional affiliations. 